\title{
Impulsive Synchronization of the New Financial Risk Dynamic System
}

\author{
Xiaoling MEI, Chengrong XIE, Yuhua XU \\ Department of Mathematics and Finance, Yunyang Teachers' College, Hubei, 442000, China
}

\begin{abstract}
In this paper, the impulsive synchronization problem of the new financial risk chaotic systems is investigated. Using the impulsive theory, A sufficient condition is derived under which the impulsively controlled the financial risk model is asymptotically stable, this condition for the stabilization of the financial risk model is less conservative. Numerical simulation example is provided to verify the effectiveness of the proposed approach.
\end{abstract}

KEYWORDS: chaotic attractor; financial risk; impulsive synchronization

\section{INTRODUCTION}

In many engineering areas, chaos theory have lots of useful applications, such as secure communication, digital communication, power electronic and power quality, biological systems, chemical reaction analysis and design, and information processing. Synchronization of chaotic systems has become one of the most interesting subjects in chaos theory [1-4]. In recent years, various effective techniques have been presented to achieve chaos synchronization [57]. Also, there are many practical examples of impulsive control systems. Three typical examples are the population control system of a kind of insects with the number of insects and their natural enemies as state variables, a chemical reactor system with the quantities of different chemicals server as the state, and a financial system with two state variables of the amount of money in a market and the saving rates of a central bank [8]. In this paper, a sufficient condition on the stability of impulsive control financial risk systems are presented, the theory of impulsive control is used to show the new financial risk model can be stabilized by discrete moments.

The new financial risk model is described by [9]

$\left\{\begin{array}{l}\dot{x}=y z-a x \\ \dot{y}=x z-b y \\ \dot{z}=c z-x y\end{array}\right.$

which $x$ describes the first stage risk of the financial system, $y$ describes the second stage risk of the financial system, $z$ describes the third stage risk of the financial system, $a, b$ and $c$ describe each stage risk intensity of the financial system, and the system (1) is chaotic for the parameters $a=5, b=9, c=1$ (see Figs.1).

For system (1), we has

$$
\nabla V=\frac{\partial \dot{x}}{\partial x}+\frac{\partial \dot{y}}{\partial y}+\frac{\partial \dot{z}}{\partial z}=c-a-b=-13<0 \text {. }
$$

Therefore, the system (1) is one dissipative system. A volume element $V_{0}$ is apparently contracted by the flow into a volume element $V_{0} \mathrm{e}^{-13 t}$ in time $\mathrm{t}$.It means that each volume containing the trajectory of this dynamical system shrinks to zero as $t \rightarrow \infty$ at an exponential rate -13 . So, all this dynamical system orbits are eventually confined to a specific subset that have zero volume, the asymptotic motion settles onto an attractor of the system(1). The Lyapunov exponent spectrum of system (1) is found to be $L_{1}=0.7680, L_{2}=-0.0056$, $L_{3}=-2.8232$ for initial value $(1,2,1)$. The Lyapunov dimension is $D_{L}=0.2700$, which means that the system (1) is really a dissipative system, and the Lyapunov dimension of this system is fractional. The fractal nature of an attractor does not merely mean this system has non-periodic orbits; it also causes nearby trajectories to diverge. Fig. 2 displays the maximum Lyapunov exponent spectrum of the system for $a \in[0,8]$. It is clear that there are some periodic windows in Fig.2. However, this periodic window is very important in the evolution of the dynamics $\operatorname{system}(1)$. 


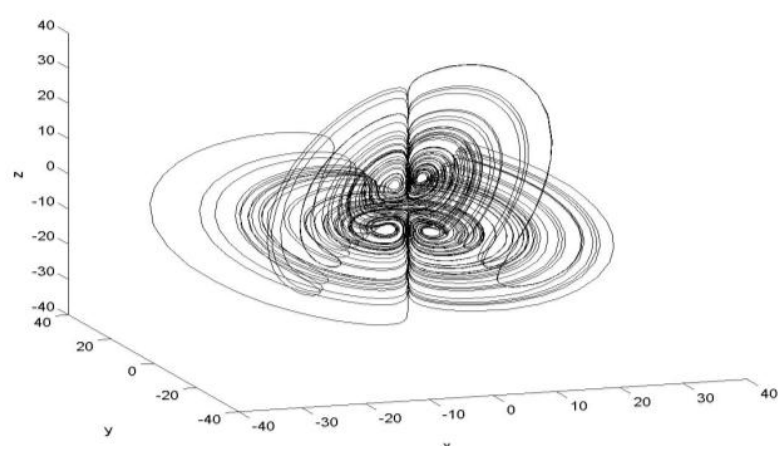

Fig.1. the new financial risk chaotic system

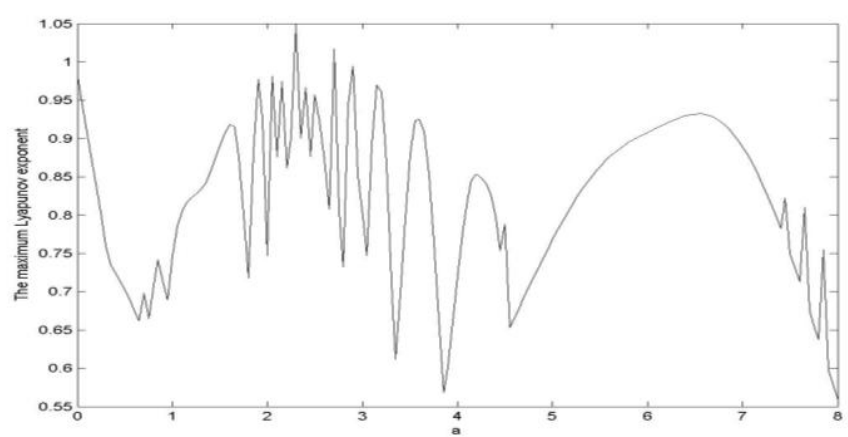

Fig.2. maximum Lyapunov exponent spectrum

$a \in[0, \mathcal{\varepsilon}$

\section{SYNCHRONIZATION OF FINANCIAL RISK DYNAMIC SYSTEM BY IMPULSIVE CONTROL}

In this section, we study the impulsive control of the new financial risk chaotic systems (1).

Let the new financial risk chaotic systems (1) as follows

$\dot{X}=\left(\begin{array}{c}\dot{x} \\ \dot{y} \\ \dot{z}\end{array}\right)=\left(\begin{array}{ccc}-a & 0 & 0 \\ 0 & -b & 0 \\ 0 & 0 & c\end{array}\right)\left(\begin{array}{l}x \\ y \\ z\end{array}\right)+\left(\begin{array}{c}y z \\ x z \\ -x y\end{array}\right)$

$\dot{X}=A X+F(X)$

where $X=\left(\begin{array}{l}x \\ y \\ z\end{array}\right), A=\left(\begin{array}{ccc}-a & 0 & 0 \\ 0 & -b & 0 \\ 0 & 0 & c\end{array}\right), F(X)=\left(\begin{array}{c}y z \\ x z \\ -x y\end{array}\right)$

Suppose that a discrete instant set $\left\{t_{k}\right\}$ satisfies $t_{0}<t_{1}<\cdots t_{k}<\cdots, \lim _{k \rightarrow \infty} t_{k}=\infty$.

Let

$\left.\Delta X\right|_{t=t_{k}}=X\left(t_{k}^{+}\right)-X\left(t_{k}\right)=I_{k}(X)$,

Where

$X\left(t_{k}^{+}\right)=\lim _{t \rightarrow t_{k}^{+}} X(t), X\left(t_{k}\right)=\lim _{t \rightarrow t_{k}^{-}} X(t)=X\left(t_{k}^{-}\right)$, then an impulsive system is given by

$$
\left\{\begin{array}{l}
\dot{X}=A X+F(t, X), \quad t \neq t_{k}, \\
\Delta X=B_{k} X, \quad t=t_{k}, \\
X\left(t_{0}^{+}\right)=X_{0}, \quad k=1,2, \ldots,
\end{array}\right.
$$

where $B_{k}$ is impulsive control matrix.

Now, we consider bidirectionally coupled impulsive control of chaotic systems in the form as follows:

$$
\begin{aligned}
& \begin{cases}\dot{X}=A X+F(t, X) & t \neq t_{k} \\
\Delta X=B_{k} e, & t=t_{k}\end{cases} \\
& \begin{cases}\dot{Y}=A Y+F(t, Y) t \neq t_{k} \\
\Delta Y=C_{k} e, & t=t_{k}\end{cases}
\end{aligned}
$$

Remark 1. In comparison with those of existing discussion, here, the two chaotic systems (3) and (4) are bidirectionally coupled different impulsive control matrix, which is different with the references [7][8][10].

Let

$$
F(t, Y)-F(t, X)=M(X, Y) e
$$

where $\|M\| \leq \lambda$.

Subtracting (3) from (4) and considering (5), we have

$\begin{cases}\dot{e}=(A+M(X, Y)) e, & t \neq t_{k}, \\ \Delta e=\left(C_{k}-B_{k}\right) e, & t=t_{k},\end{cases}$

where

$e=\left(e_{1}, e_{2}, \ldots, e_{n}\right)^{T}=\left(Y_{1}-X_{1}, Y_{2}-X_{2}, \ldots, Y_{n}-X_{n}\right)^{T}$

The objective is to find some conditions on the impulsive control matrix $C_{k}-B_{k}$ and the impulsive distances $t_{k}-t_{k-1}, \quad k=1,2, \ldots, \quad$ such that the impulsive error system (6) is asymptotical stable at origin.

Theorem 1. The systems (3) and (4) can realize impulsive synchronization using the following form

$$
\ln \theta \eta_{k}+\mu\left(t_{k}-t_{k-1}\right) \leq 0,
$$

where $\eta_{k}=\lambda_{\text {max }}\left[\left(I+C_{k}-B_{k}\right)^{T}\left(I+C_{k}-B_{k}\right)\right]$,

$\theta \geq 1, \mu \geq\|A+M\|$.

Proof: Let the candidate Lyapunov function be in the form of $V(e)=\frac{1}{2} e^{T} e$.

The time derivative along the trajectory (6) is $\dot{V}(e)=e^{T} \dot{e}=e^{T}(A+M) e \leq\left|e^{T}\right||A+M \| e| \leq \mu V(e)$. 
This implies that

$V(e(t)) \leq V\left(e\left(t_{k-1}^{+}\right)\right) \exp \left(\mu\left(t-t_{k-1}\right)\right), t \in\left(t_{k-1}, t_{k}\right]$, $k=1,2, \cdots$.

Now from (6), we have

$$
\begin{aligned}
V\left(e\left(t_{k}^{+}\right)\right) & =0.5\left[\left(I+C_{k}-B_{k}\right) e\right]^{T}\left(I+C_{k}-B_{k}\right) e \\
& =0.5 e^{T}\left(I+C_{k}-B_{k}\right)^{T}\left(I+C_{k}-B_{k}\right) e \\
& \leq \lambda_{\max }\left[\left(I+C_{k}-B_{k}\right)^{T}\left(I+C_{k}-B_{k}\right)\right] 0.5 e^{T} e \\
& \leq \lambda_{\text {ma }}\left[\left(I+C_{k}-B_{k}^{T}\right) I\left(+C_{k}-B_{k}\right)\right] e^{\Phi} e \\
& =\eta_{k} 0.5 e^{T} e=\eta_{k} V\left(e\left(t_{k}\right)\right) .
\end{aligned}
$$

When $t \in\left(t_{0}, t_{1}\right]$,

$V(e(t)) \leq V\left(e\left(t_{0}^{+}\right)\right) \exp \left(\mu\left(t-t_{0}\right)\right)$, then

$V\left(e\left(t_{1}\right)\right) \leq V\left(e\left(t_{0}^{+}\right)\right) \exp \left(\mu\left(t_{1}-t_{0}\right)\right)$.

So,

$V\left(e\left(t_{1}^{+}\right)\right) \leq \eta_{1} V\left(e\left(t_{1}\right)\right) \leq \eta_{1} V\left(e\left(t_{0}^{+}\right)\right) \exp \left(\mu\left(t_{1}-t_{0}\right)\right)$.

In the same way for $t \in\left(t_{1}, t_{2}\right]$, we have

$V(e(t)) \leq V\left(e\left(t_{1}^{+}\right)\right) \exp \left(\mu\left(t-t_{1}\right)\right)$

$\leq \eta_{1} V\left(e\left(t_{0}^{+}\right)\right) \exp \left(\mu\left(t-t_{0}\right)\right)$

So, $\forall t \in\left(t_{k}, t_{k+1}\right]$,

$V(e(t)) \leq V\left(e\left(t_{0}^{+}\right)\right) \eta_{1} \eta_{2} \cdots \eta_{k} \exp \left(\mu\left(t-t_{0}\right)\right)$.

Thus, $\quad \forall t \in\left(t_{k}, t_{k+1}\right], k=1,2, \ldots$,

$V(e(t)) \leq V\left(e\left(t_{0}^{+}\right)\right) \eta_{1} \eta_{2} \cdots \eta_{k} \exp \left(\mu\left(t-t_{0}\right)\right)$

$\leq V\left(e\left(t_{0}^{+}\right)\right) \eta_{1} \eta_{2} \cdots \eta_{k} \exp \left(\mu\left(t_{k+1}-t_{0}\right)\right)$

$=V\left(e\left(t_{0}^{+}\right)\right) \eta_{1} \exp \left(\mu\left(t_{2}-t_{1}\right)\right) \eta_{2} \exp \left(\mu\left(t_{3}-t_{2}\right)\right)$

$\cdots \eta_{k} \exp \left(\mu\left(t_{k+1}-t_{k}\right)\right) \exp \left(\mu\left(t-t_{0}\right)\right)$

From the assumptions given in the theorem

$\eta_{k} \exp \left(\mu\left(t_{k+1}-t_{k}\right)\right) \leq \frac{1}{\theta}, \quad k=1,2, \cdots$,

We have $V(e(t)) \leq V\left(e\left(t_{0}^{+}\right)\right) \frac{1}{\theta^{k}} \exp \left(\mu\left(t-t_{0}\right)\right)$.

That is

$$
V(e(t)) \leq V\left(e\left(t_{0}^{+}\right)\right) \frac{1}{\theta^{k}} \exp \left(\mu\left(t-t_{0}\right)\right), t \geq t_{0} .
$$

From Ref. [10], this implies that the origin in system (6) is globally asymptotically stable or the slave system is synchronized with the master system asymptotically for any initial conditions.

\section{SIMULATION AND RESULTS}

In simulations, the initial conditions of the master and slave systems are $(1,1,1)$ and $(1,1,1)$, respectively.. We choose the gain matrices $C_{k}=3 B_{k}, \quad B_{k}(k=1,2, \ldots)$ is a constant matrix $B_{k}=\operatorname{diag}(-0.5,-0.6,-0.7)$, then $\eta_{k}=0.16$. Let $\theta=1.5$, from $\ln \theta \eta_{k}+\mu\left(t_{k}-t_{k-1}\right) \leq 0$, then the solutions $t_{k}-t_{k-1} \leq 0.0408$ are obtained for $\mu=35$, we let $t_{k}-t_{k-1}=0.04$.We introduce the quantity $E(t)=\sqrt{e_{1}^{2}+e_{2}^{2}+e_{3}^{2}}$, which is used to measure the quality of the control process. It is obvious that when $E(t)$ no longer increases, two chaotic systems achieve synchronization. In Fig.3, three state errors versus time are shown and the state errors tend to zero asymptotically as time evoles.
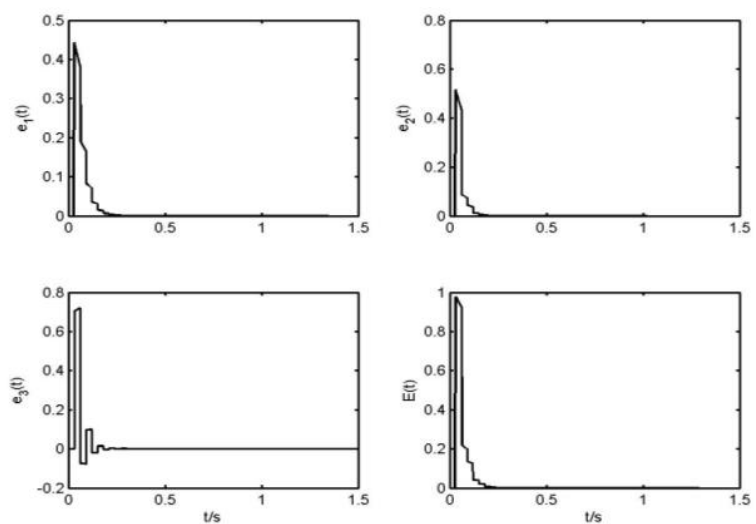

Fig.3. Synchronization errors with time $t$

\section{CONCLUSION}

In this paper, we use impulsive control theory to impulsively control the new financial risk chaotic systems, sufficient conditions are derived for the synchronization of the new chaotic systems, numerical simulations are then given to verify the effectiveness of the proposed schemes.

\section{ACKNOWLEDGEMENTS}

This research is supported by the Youth Fund Project of the Humanities and Social Science Research for the Ministry of Education of China (14YJCZH173), the Science and Technology Research Youth Project for the Education Department of Hubei Province of China (Q20145001). 


\section{REFERENCES}

[1] C. Sparrow.1982.The Lorenz equations: Bifurcation, Chaos, and Strange Attractors, New York: Springer.

[2] J. Lü, A. Lu, G. Chen. 2002. Chaotic Time Series Analysis and its Application, Wuhan: Wuhan University Press.

[3] Y. Wang, Z. Guan, H. Wang.2003.Feedback an adaptive control for the synchronization of Chen system via a single variable. Physics Letter A, 312, 34-40.

[4] J. Lü, A. Lu.2003.Controlling uncertain Lü system using linear feedback, Chaos, Solitons \& Fractals, 17, 127-33.

[5] G. Chen, X. Dong.1998.From Chaos to Order: Methodologies, Perspectives, and Applications, Singapore: World Scientific Pub. Co.
[6] E. M. Elabbssy, H. N. Agiza, M. M. ElDessoky.2006.Adaptive synchronization of a hyperchaotic system with uncertain parameter, Chaos, Solitons \& Fractals,30, 1133-1142.

[7] Y. Tang, W. Wong, J. Fang, Q. Miao. 2011.Pinning impulsive synchronization of stochastic delayed coupled networks. Chin. Phys. B, 20,040513(1-10)

[8] J. Sun, F. Qiao, Q. Wu. 2005. Impulsive control of a financial model, Physics Letters A ,335, 282-288.

[9] Y. Xu, C. Xie, Y. Wang.2015. Research on the evolution mechanism of the financial risk system, Statistics and Decision, accepted.

[10] T. Yang.2001.Impulsive Control Theory. Berlin: Springer. 